ich mich z. B. in Wernigerode am Harz persönlich überzeugt habe.

Eine Vergleichung, die Hellmann zwischen Berlin, Innenstadt und der Außenstation in der Seestraße macht ${ }^{1}$ ), gibt, obwohl sie bereits ein Charakteristikum des Stadteinflusses, die bedeutende Abstumpfung extremer Strahlungskฌlte erkennen läßt, doch noch um die Hälfte zu kleine Werte für den Gesamtbetrag des Berliner Stadteinflusses. Dieser reicht eben heutzutage doch um mehrere, etwa $10 \mathrm{~km}$ über das Weichbild der Hauptstadt hinaus, was sich wegen des fast gänzlichen Feblens der in der weiteren Umgebung alljährlich auftretenden Frühjahrsfröste, insbesondere in phänologischer Beziehung, sehr auffállig bemerkbar macht. Der Frühling tritt in Berlin 8-14 Tage früher ein als sonst in der Mark Brandenburg.

5 5. Zusammenfassung. Die Resultate vorliegender Arbeit lassen sich somit in folgenden Sätzen zusammenfassen : I. Temperaturbeobachtungen, die um Sonnenaufgang,
Mittag und Sonnenuntergang angestellt sind, lassen sich mit einer 0.2 höchst selten übersteigenden Unsicherheit für den Einzelfall auf wahre Monatsmittel reduzieren.

2. Die von Kirch angestellten Beobachtungen in der ersten Hälfte des 18 . Jahrhunderts sind höchst wahrscheinlich in dieser Art angestellt. Eine Reduktion derselben gibt durchaus. als vertrauenswürdig anzusehende Werte.

3. Die Kirchschen Beobachtungen sind die einzigen vom Stadteinfiuß freien unter allen Berliner Beobachtungsreiben ${ }^{2}$ ).

4. Aus ihnen geht hervor, daß sich das Klima der Mark Brandenburg seit 200 Jahren nicht im geringsten geändert hat. Dagegen können Epochen von 20-30 Jahren eintreten vielleicht mit den Briucknerschen identisch - innerhalb deren das Gesamtmittel der Temperatur um $1 / 2{ }^{\circ} \mathrm{C}$. über oder unter dem Säkularmittel liegt. (Die Epoche 1730-1750 war trotz des abnorm kalten Jahres 1740 von $5: 2$ red. normal warm.) Potsdam, Igis Aug. I4.

O. Meißner.

1) Klima von Berlin, II. Teil, S. 30 u. 46.

2) Die Sternwartenbeobachtungen $1841-1847$ hat Hellmann auf die anderen Reihen reduziert und damit natürlich den Stadteinfluß, soweit er nicht an sich schon darin steckte, mit hineingebracht.

\title{
Mitteilungen über Kleine Planeten.
}

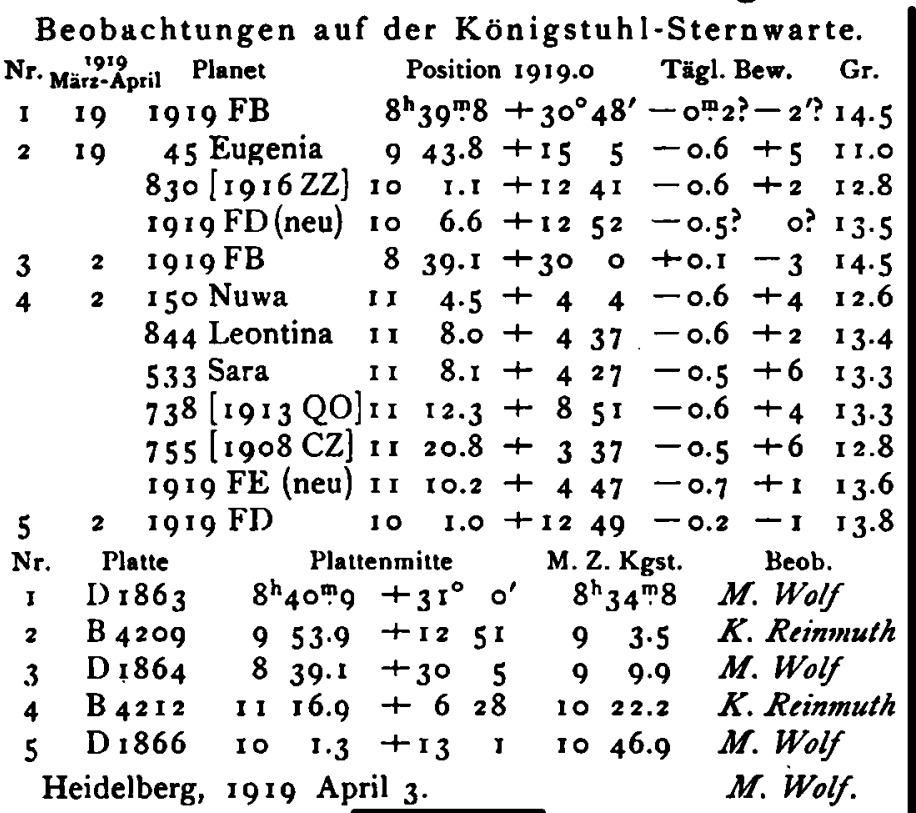

Beobachtungen auf der Universitätssternw. Wien. Planet 1919 M. Z. Wien Scheinb. Ort 844 Leontina März 3 I $3^{\mathrm{h}} 29^{\mathrm{m}} 5 \mathrm{I}^{\mathrm{s}}$ I $1^{\mathrm{h}} 30^{\mathrm{m}} 17^{\mathrm{s}} 47$ + $3^{\circ}$ I $5^{\prime} 3^{8 .} \cdot 3$

\begin{tabular}{|c|c|c|c|}
\hline 81 Terpsichore & $\begin{array}{c}1929 \\
\text { März } 6\end{array}$ & $\begin{array}{l}\text { M.E.Z. } \\
9^{\text {h }} 33^{\mathrm{m}}\end{array}$ & $\begin{array}{l}\text { Position 1919.0 } \\
9^{\text {h }} 34^{\mathrm{m}} 19^{\mathrm{s}}+21^{\circ} 28 \text { ! }^{\prime} \mathrm{I}\end{array}$ \\
\hline 799 [I9 Is IVO] & Mărz 7 & 1053 & $\begin{array}{c}1053.57+651.1 \\
7 . \text { Rheden. }\end{array}$ \\
\hline
\end{tabular}

Beobachtungen auf der Fabra-Stérnwarte.

\begin{tabular}{|c|c|c|c|c|}
\hline $\begin{array}{l}\text { Planète } \\
9 \text { Fortuna }\end{array}$ & $\begin{array}{c}1919 \\
\text { Févr. } 20\end{array}$ & $\begin{array}{l}\text { T. m. Gr. } \\
\text { I } 2^{h} 0^{m}\end{array}$ & $\begin{array}{l}\text { Position } \\
\text { Ioh } 3^{8^{m}}+8^{s}\end{array}$ & $\begin{array}{l}1919.0 \\
+6^{\circ} 24 ! 4\end{array}$ \\
\hline Amphitrite & , 26 & 120 & 1055 & +955.4 \\
\hline Tacuia & 20 & 120 & $10 \quad 4^{8} 50$ & + \\
\hline
\end{tabular}

Planète 1919 T.m.Gr. Position 1919.0 349 Dembowska Févr. 26 I $2^{\mathrm{h}} \mathrm{O}^{\mathrm{m}}$ I $\mathrm{I}^{\mathrm{h}} \mathrm{II}^{\mathrm{m}} 59^{\mathrm{s}}+15^{\circ} 29^{\prime} 4$ 367 Amicitia $》 26$ I2 0 II $016+1229.2$ Barcelone, 1919 Mars II. F. Comas Sold.

Planet igi7a.

Im Journal des Observateurs II, Nr. 9, S. I I 5, werden von $F$. Sy die Ausmessangen der Aufnahmen des Planeten r9 7 a $=$ Alger r917 C (s. A. N. 208.44) veröffentlicht. .Aus den Beobachtungen 1917 Dez. I I, 22 und 1918 Jan. 3 habe ich folgendes Elementensystem berechnet:

$$
\begin{aligned}
& \text { I9I } 7 \text { Dez. } 3 \text { I.0 m. Z. Greenwich. } \\
& M=331^{\circ} 16^{\prime} 34^{\prime} .9 \\
& \omega=87373^{8.5} \\
& \delta=372454.6 \\
& i=2128.4 \\
& \begin{aligned}
\varphi & =17^{\circ} 19^{\prime} 32^{\prime} 1 \\
\mu & =591.788 \\
\operatorname{og} a & =0.518561
\end{aligned}
\end{aligned}
$$

welches in den beiden einzigen, noch weiter vorliegenden $\mathrm{Be}$ obachtungen die folgenden Fehler läßt: Dez. $12 A \lambda=-1: 4$,

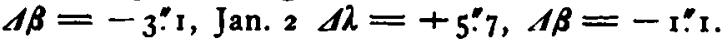

Der Planet ist besonders auffallend wegen seiner mittleren

\begin{tabular}{|c|c|c|c|c|c|}
\hline \multicolumn{2}{|c|}{1919} & \multirow{2}{*}{$\begin{array}{l}\alpha_{1919.0} \\
I^{\mathrm{h}} 47^{\mathrm{m}} \cdot 3\end{array}$} & \multicolumn{2}{|c|}{81919.0} & \multirow{2}{*}{$\begin{array}{r}\log \Delta(r) \\
\left(0.45^{2}\right)\end{array}$} \\
\hline April & 3 & & $-10^{\circ}$ & 11 & \\
\hline & II & 41.1 & 9 & $4 I$ & \\
\hline & 19 & & $y$ & I I & 0.273 \\
\hline & 27 & & 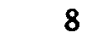 & 42 & \\
\hline Mai & 5 & 0 & 8 & 17 & 0.29 \\
\hline & 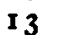 & 18.9 & 7 & $5^{8}$ & $(0.47$ \\
\hline
\end{tabular}
Bewegung und der dafür ungewöhnlichen Exzentrizität.

Zur dringend erwünschten Wiederauffindung in der kurz bevorstehenden Opposition - in den nächsten Jahren würde er außerst lichtschwach sein - diene die nachstehende Ephemeride ( $12^{h} \mathrm{~m}$. Z. Gr.):

Opposition April 16. Größe I $3^{\mathrm{m}} 7 . \quad M=49^{\circ}$. Berlin-Dahlem, Astr. Rechen-Institut, 19 I 9 März 25. W. Strehlow.

In halt ZU Nr. 4983. H.E. Lau †. Beobachtungen des Planeten Mars. 217. - P. Guthnick. Veränderlichkeit von 12 Lacertae. 219. - O. Meißner. Reduktion der Kirchschen Temperaturbeobachtungen in Berlin (1719-1751). 225. - Mitteilungen uber Kleine Planeten. 231.

Geachlowen 1939 April 12. Hernusgeber: H. Kobold. Druck ros C. Schaidt. Lxpedition: Kiel, Moltkeatr. 8o. Postucheck-Konto Nr. 6z38 Hamburg 1x. 